\title{
Ultrasonographic findings in a patient with reactive arthritis induced by intravesical BCG therapy for bladder cancer
}

Hitoshi Yoshimura, Tadashi Okano, Kentaro Inui, Hiroaki Nakamura

\begin{tabular}{|c|l|}
\hline Citation & Journal of Medical Ultrasonics. 46(1); 163-165 \\
\hline Issue Date & $2019-01-25$ \\
\hline Type & Journal Article \\
\hline Textversion & Author \\
\hline Relation & $\begin{array}{l}\text { This is a post-peer-review, pre-copyedit version of an article published in Journal } \\
\text { of Medical Ultrasonics. The final authenticated version is available online at: } \\
\text { https://doi.org/10.1007/s10396-018-0889-7. }\end{array}$ \\
\hline DOI & $10.1007 / \mathrm{s} 10396-018-0889-7$ \\
\hline
\end{tabular}

\author{
Self-Archiving by Author(s) \\ Placed on: Osaka City University
}

Yoshimura, H., Okano, T., Inui, K. et al. Ultrasonographic findings in a patient with reactive arthritis induced by intravesical BCG therapy for bladder cancer. Journal of Medical Ultrasonics. 46, 163-165 (2019). Doi:10.1007/s10396-018-0889-7 


\title{
Ultrasonographic findings in a patient with reactive arthritis induced by intravesical BCG therapy for bladder cancer
}

\section{Hitoshi Yoshimura, Tadashi Okano, Kentaro Inui, Hiroaki Nakamura}

Department of Orthopaedic Surgery, Osaka City University Graduate School of Medicine, Osaka, Japan

Correspondence to: Tadashi Okano, Department of Orthopaedic Surgery, Osaka City University Graduate School of Medicine, 1-4-3 Asahimachi, Abeno-ku, Osaka 545-8585, Japan

E-mail:ma1sa3ru@med.osaka-cu.ac.jp

\section{Key words}

ultrasonography, enthesitis, reactive arthritis, spondyloarthritis, BCG therapy

\begin{abstract}
Intravesical bacillus Chalmette-Guerin (BCG) therapy is an effective and safe immunotherapy for superficial bladder cancer. However, reactive arthritis $(\operatorname{Re} A)$ is reported as an adverse event in about $0.5-1 \%$ of patients receiving intravesical BCG therapy. ReA is known as a kind of spondyloarthritis, the main pathological finding of which is enthesitis. Here we report a patient with ReA induced by intravesical BCG therapy for bladder cancer, in whom the diagnosis of ReA was supported by ultrasonographic findings of enthesitis and synovitis. A 69-year-old male was diagnosed with carcinoma in situ of the bladder and treated with intravesical BCG therapy. After the third intravesical BCG injection, pain and swelling appeared in both wrists. Ultrasonographic examination revealed not only intra-articular synovitis in the bilateral wrist joint, wrist flexor tenosynovitis, and wrist extensor tenosynovitis but also enthesitis of the flexor carpi radialis tendon (FCR). The severe enthesitis of the FCR in both wrists was considered to be an important finding that supported the diagnosis in this patient. Thus, we diagnosed this case as ReA induced by intravesical BCG therapy. In clinical practice, $\operatorname{ReA}$ is often difficult to distinguish from seronegative rheumatoid arthritis and other rheumatic diseases. Ultrasonographic findings of enthesitis may support a definitive diagnosis for these patients.
\end{abstract}




\section{Introduction}

Intravesical bacillus Chalmette-Guerin (BCG) therapy is an effective and safe immunotherapy for superficial bladder cancer. However, reactive arthritis $(\operatorname{Re} A)$ is reported as an adverse event in about $0.5-1 \%$ of patients receiving intravesical BCG therapy $[1,2]$. $\operatorname{ReA}$ is known as a kind of spondyloarthritis ( $\mathrm{SpA})$, the main pathological finding of which is enthesitis. In clinical practice, $\operatorname{ReA}$ is often difficult to distinguish from seronegative rheumatoid arthritis and other rheumatic diseases. Recently, high-resolution ultrasonography has become a useful tool for diagnosing rheumatic diseases $[3,4]$. Currently, we can distinguish various pathological inflammatory findings such as intra-articular synovitis, tenosynovitis, and enthesitis by using ultrasonography [5]. Here we report a patient with ReA induced by intravesical BCG therapy for bladder cancer, in whom the diagnosis of ReA was supported by ultrasonographic findings of enthesitis and synovitis.

\section{Case report}

A 69-year-old male was diagnosed with carcinoma in situ (CIS) of the bladder and treated with intravesical BCG therapy. After the third intravesical BCG injection, pain and swelling appeared in the left wrist joint. Over the following weeks, symptoms became worse and pain and swelling also appeared in the right wrist. Blood examination showed inflammatory markers to be elevated: white blood cell count $(6000$ cells $/ \mu \mathrm{L}), \mathrm{C}$ reactive protein $(3.57$ $\mathrm{mg} / \mathrm{dL}$ ), matrix metalloproteinase-3 $(128.6 \mathrm{ng} / \mathrm{mL}$ ). Immunological (rheumatoid factor, anticyclic citrullinated peptide antibody and anti-nuclear antibodies) and infectious disease tests (hepatitis virus $B$ and $C$, tuberculosis TNFY, $\beta$-D-glucan) were all negative. Human leukocyte antigen (HLA) typing revealed the presence of A2, A26, B35, B54, Cw1, Cw9, DR4, DQ4, and DQ8. Ultrasonographic examination found not only intra-articular synovitis in the bilateral wrist joints, wrist flexor tenosynovitis, and wrist extensor tenosynovitis but also enthesitis of the flexor carpi radialis tendon (FCR) (Fig. 1, left). The severe enthesitis of the FCR in both wrists was considered to be an important finding that supported the diagnosis in this patient. Thus, we diagnosed this case as ReA induced by intravesical BCG therapy. Symptoms did not improve on NSAID therapy, so methotrexate, salazosulfapyridine, and prednisolone were administered. After 1 month, the wrist pain and swelling had reduced and the ultrasonographic inflammatory findings had also improved (Fig. 1, middle). During ReA treatment, intravesical BCG injection was continued, and all intravesical injections were completed about 3 months later. After 6 months, the symptoms and ultrasonographic inflammatory findings had almost disappeared (Fig. 1, right). 


\section{Discussion}

According to a previous report by Bernini et al., about $55 \%$ of patients with $\operatorname{ReA}$ due to intravesical BCG therapy showed polyarthritis and $37 \%$ had oligoarthritis [2]. The affected joints were knee $(84.3 \%)$, ankle $(55.1 \%)$, hand $(39.3 \%)$, and wrist $(32.6 \%)$. The relationship between HLA-B27 and ReA is well known, and the positivity ratio of HLA-B27 in patients with ReA was $42.6 \%$. A recent study showed that not only HLA-B27 but also B35, B39, and B51 positivity were more frequent in ReA induced by intravesical BCG therapy [6]. In the present case, however, bilateral wrist arthritis, indicating oligoarthritis, was observed and HLA-B27 was negative, but B35 was positive. Although these clinical and laboratory findings were not contradictory for the diagnosis of ReA due to intravesical BCG therapy, it was considered to be insufficient evidence for a definitive diagnosis. However, when the ultrasonographic examination revealed enthesitis of the FCR together with intra-articular synovitis and tenosynovitis, these findings confirmed the diagnosis of ReA. Practically, typical enthesitis, such as enthesis of the Achilles tendon or lateral epicondyle and so on, is easy to identify by physical examination. However, enthesitis with intra-articular synovitis in small joints is usually difficult to distinguish upon physical examination. Enthesitis is the main characteristic of SpA including ReA, and it is important for distinguishing SpA from RA [7]. Using ultrasonography, we can detect the location of inflammation, and therefore, distinguish between intra-articular synovitis, tenosynovitis, and enthesitis. There has been just one report describing ultrasonographic findings of ReA due to intravesical BCG therapy, but ultrasonographic images were not presented [8]. This is the first report showing ultrasonographic enthesitis images in a patient with ReA induced by intravesical BCG therapy for bladder cancer.

In conclusion, we have demonstrated that patients with ReA induced by intravesical BCG therapy can be definitively diagnosed using ultrasonographic imaging of enthesitis. Intravesical BCG therapy has recently become a common treatment for superficial bladder cancer, and ReA due to intravesical BCG therapy should be considered one of the adverse events. Ultrasonographic findings of enthesitis may support a definitive diagnosis for these patients.

Disclosure statement: The authors have declared no conflicts of interest.

Funding: No specific funding was received to carry out the work described in this manuscript. 
Ethical statements: All procedures followed were in accordance with the ethical standards of the responsible committee on human experimentation (institutional and national) and with the Helsinki Declaration of 1964 and later versions. Informed consent was obtained from the patient for being included in the study.

\section{References}

1 Lamm DL, Stogdill VD, Stogdill BJ, et al. Complications of BCG immunotherapy in 1278 patients with bladder cancer. J Urol. 1986 2;135:272-4.

2 Bernini L, Manzini CU, Giuggioli D, et al. Reactive arthritis induced by intravesical BCG therapy for bladder cancer: our clinical experience and systematic review of the literature. Autoimmun Rev. 2013;12:1150-9.

3 Grassi W, Okano T, Di Geso L, et al. Imaging in rheumatoid arthritis: options, uses and optimization. Expert Rev Clin Immunol. 2015;11:1131-46.

4 Grassi W, Okano T, Filippucci E. Use of ultrasound for diagnosis and monitoring of outcomes in crystal arthropathies. Curr Opin Rheumatol. 2015;27:147-55.

5 Klocke R. Ultrasound and the clinical rheumatologist. Rheumatology. 2013;52:1151-2.

6 Taniguchi $\mathrm{Y}$, Nishikawa H, Karashima T, et al. Frequency of reactive arthritis, uveitis, and conjunctivitis in Japanese patients with bladder cancer following intravesical BCG therapy: A 20-year, two-centre retrospective study. Joint Bone Spine. 2017;84:637-8.

7 Taniguchi $\mathrm{Y}$, Kumon Y, Ohnishi T, et al. Frequency of enthesitis in apparently healthy Japanese subjects detected by 18F FDG-PET/CT. Mod Rheumatol. 2012;22:939-41.

8 Ben Abdelghani K, Fazaa A, Souabni L, et al. Reactive arthritis induced by intravesical BCG therapy for bladder cancer. BMJ Case Rep. 2014. doi:10.1136/bcr-2013-202741.

\section{Figure legend}

Fig. 1. Ultrasonographic findings

Not only intra-articular synovitis in the bilateral wrist joint, wrist flexor tenosynovitis, and wrist extensor tenosynovitis but also enthesitis of the flexor carpi radialis tendon (FCR) were observed (left). After 1 month, the ultrasonographic inflammatory findings had improved (middle). After 6 months, the ultrasonographic inflammatory findings had almost disappeared (right). These images were captured at the most severe inflammatory site in the same joint. 


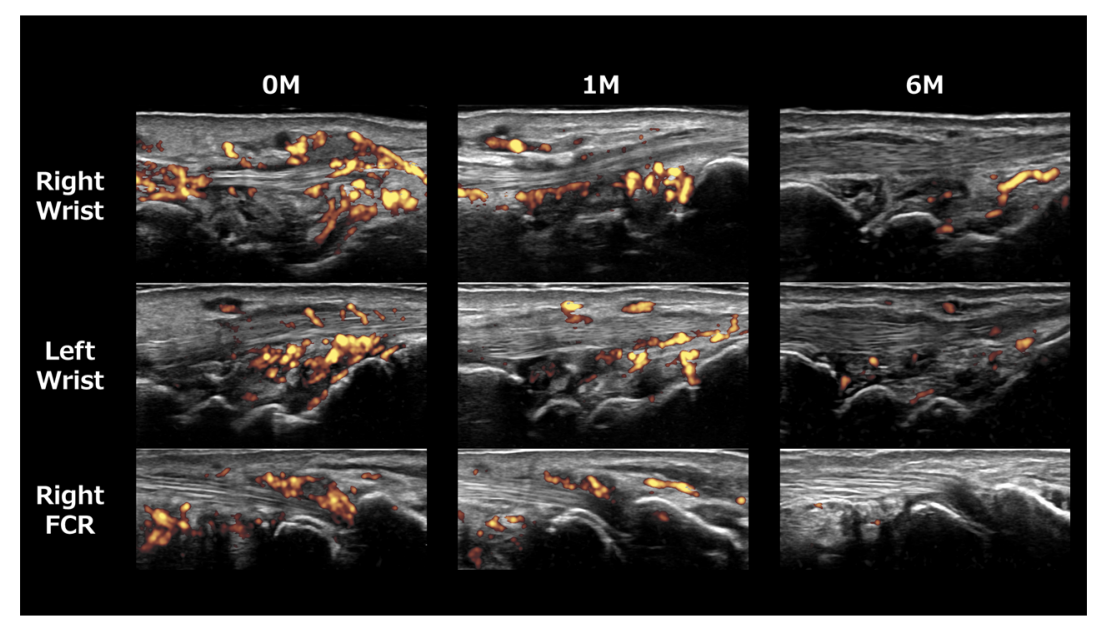

